\title{
Adapting technology-based HIV prevention and care interventions for youth: lessons learned across five U.S. Adolescent Trials Network studies
}

\author{
Danielle Giovenco $^{1}$, Kathryn E. Muessig ${ }^{2}$, Casey Horvitz ${ }^{3}$, Katie B. Biello ${ }^{4,5}$, Albert Y. Liu ${ }^{6}$, Keith J. Horvath ${ }^{7}$, \\ Jesse M. Golinkoff ${ }^{8}$, Cathy J. Reback ${ }^{9}$, Lisa Hightow-Weidman ${ }^{2,3}$
}

${ }^{1}$ Department of Epidemiology, ${ }^{2}$ Department of Health Behavior, ${ }^{3}$ Institute for Global Health and Infectious Diseases, University of North Carolina at Chapel Hill, Chapel Hill, NC, USA; ${ }^{4}$ Departments of Behavioral \& Social Sciences and Epidemiology, Center for Health Equity Research, Brown University, Providence, RI, USA; ${ }^{5}$ The Fenway Institute, Fenway Health, Boston, MA, USA; ${ }^{6}$ San Francisco Department of Public Health, Bridge HIV, San Francisco, CA, USA; ${ }^{7}$ Department of Psychology, San Diego State University, San Diego, CA, USA; ${ }^{8}$ School of Nursing, University of Pennsylvania, Philadelphia, PA, USA; ${ }^{9}$ Friends Research Institute, Los Angeles, CA, USA

Contributions: (I) Conception and design: D Giovenco, KE Muessig, C Horvitz, L Hightow-Weidman; (II) Administrative support: KE Muessig, L Hightow-Weidman; (III) Provision of study materials or patients: All authors; (IV) Collection and assembly of data: All authors; (V) Data analysis and interpretation: D Giovenco, KE Muessig, C Horvitz; (VI) Manuscript writing and revisions: All authors; (VII) Final approval of manuscript: All authors.

Correspondence to: Danielle Giovenco. 135 Dauer Drive, 2101 McGavran-Greenberg Hall, CB \#7435, Chapel Hill, NC 27599-7435, USA.

Email: dgiovenco@unc.edu; daniellegioven@gmail.com.

Background: U.S. sexual and gender minority youth experience individual, interpersonal, and structurallevel barriers to HIV prevention and care. Innovative, youth-driven approaches to mobile and electronic interventions that support use of new biomedical prevention, testing, and treatment options may address these barriers. Adapting evidence-based interventions for youth must balance core intervention components with responsiveness to the distinct needs of end-users.

Methods: The UNC/Emory Center for Innovative Technology (iTech) adapts and evaluates technologybased interventions for youth living with or at risk for HIV. We analyzed formative research (focus groups and individual usability sessions) across five iTech studies: two apps promoting HIV testing and pre-exposure prophylaxis (PrEP), one app promoting behavioral risk reduction and PrEP, one PrEP adherence app, and one mobile-optimized website for increasing viral suppression, with the aim of informing best practices for technology-based intervention development. Each study presented prototypes of adapted mHealth interventions to samples of their target end-user population for use and/or evaluation.

Results: One hundred and thirty-eight youth across seven geographically diverse sites provided feedback during the intervention adaptation process. We found high interest in and acceptability of all five intervention prototypes. Cross-study themes included: (I) Desire for multiple privacy protections (e.g., password, fingerprint) to keep HIV status, sexual identity, and sexual behavior confidential. (II) Strong but varied preferences for the look and feel of platforms. Imagery should be discrete but representative. Participants valued customizable platforms and positive themes, motivational language, and humor. Youth wanted information presented using multiple modalities (e.g., text, video, image) to increase engagement. (III) Youth preferred engagement features and functions consistent with familiar platforms (e.g., Snapchat, Instagram). Gamification features that resulted in tangible versus virtual rewards were predicted to increase engagement. Intervention messaging functions were perceived as useful; customization was desired as a way to control frequency, mode (e.g., SMS, in-app message, push notification), and content. (IV) Youth voiced varied preferences for platform content including: featuring young role models from the lesbian, gay, bisexual, transgender, queer and/or questioning (LGBTQ) community, incorporating mental health resources, and maintaining a holistic health-focus (not HIV-centric).

Conclusions: We found high acceptability and consistent feedback in youths' evaluations of these mHealth 
interventions; divergence was most commonly found in preferred content versus features and functions. Identifying broadly accepted aspects of mHealth interventions for youth supports the feasibility of adaptation (versus de novo creation) and should guide the focus of future formative research phases. Continued research is needed to better understand how to balance usability preferences with finite resources for customization.

Keywords: HIV; youth; adolescent; intervention; mHealth

Received: 17 January 2020; Accepted: 18 June 2020; Published: 20 April 2021.

doi: $10.21037 /$ mhealth-20-43

View this article at: http://dx.doi.org/10.21037/mhealth-20-43

\section{Introduction}

In the United States, youth aged 13-24 years are disproportionately affected by HIV, accounting for $21 \%$ of all new HIV diagnoses (1). Young people are less likely to be aware of their infection compared to adults (2) and are less likely than adults to use pre-exposure prophylaxis (PrEP) to prevent HIV (3). Further, youth with HIV are the least likely of any age group to be linked to care in a timely manner, to take antiretroviral therapy (ART), and have a suppressed viral load (4-6). Youth face numerous barriers, including low sexual health literacy and limited access to youth-friendly and confidential HIV services, which contribute to disparities in prevention and care program effectiveness (6-9). The UNC/Emory Center for Innovative Technology (iTech) develops and tests technology-based interventions across the prevention and care continua for youth at-risk for, or living with, HIV across the United States with the goal of decreasing the impact of HIV on the lives of adolescents and young adults (10).

The use of information and communication technologies for health, or eHealth, is a key means of reaching and engaging youth in public health interventions. Mobile health (mHealth) interventions in particular offer highly accessible and adaptable platforms-including smartphone applications, mobile-optimized websites, text messaging, and social media-integrated interventions-for targeting key barriers to HIV prevention and care service utilization among youth. Estimates from 2019 show that $96 \%$ of young people aged 18-29 years in the United States report using smartphones, $90 \%$ report using at least one social media site, and $100 \%$ report using the internet (11). Research has demonstrated the potential impact of mHealth interventions for youth across the HIV prevention and care continua, ranging from biomedical prevention to testing and treatment (12-14).

The impact of mHealth interventions depends on successful identification of the health needs of end users and the ability of the platform, in design and delivery, to address multi-level barriers that would prevent the intervention from serving those needs (15). Formative evaluations of mHealth interventions often precede intervention trials or implementation to identify user preferences surrounding platform access, functionality, usability, content, and a variety of social and behavioral factors that may impact uptake and sustained use. These formative efforts are designed to optimize an intervention to a specific group of users to maximize engagement. By harnessing lessons learned from formative work across mHealth research, we can identify consistencies in intervention preferences among users. We aim to lessen the burden of future formative investigations and identify cross-population differences, indicating where targeted formative work may be needed for intervention tailoring.

We conducted a summative evaluation of formative research across five iTech-supported studies in the Adolescent Medicine Trials Network for HIV/AIDS Interventions (ATN), each of which produced functional prototypes of mHealth primary and secondary HIV prevention interventions that were used and evaluated by sexual and gender minority youth. Our objective was to create a toolbox for researchers aiming to adapt mHealth interventions to best serve youth and inform a best practices model for technology-based intervention development. Similarities in intervention preferences across studies were identified, as well as divergence in preferences, where formative work may still be warranted.

\section{Methods}

\section{Study settings}

iTech's formative focus groups and usability sessions were conducted in Houston, TX, Los Angeles, CA, New 
York City, NY, Philadelphia, PA, Chicago, IL, Tampa, FL, and Boston, MA. Focus groups for MyChoices (16), YouTHrive (17), LYNX (18), and TechStep (19) were conducted at study recruitment venues, usually a community clinic or health center. P3 (20) conducted individual usability sessions in a clinic. All focus groups and usability sessions were conducted in person except for LYNX's second round of focus groups, which were conducted by a project coordinator at the study venue over video chat with the study team. Participants were compensated for their time and travel with gift cards or cash and refreshments.

\section{Recruitment, eligibility, and enrollment}

Participants were recruited through a variety of strategies including social media ads, flyers, palm cards, and inperson recruitment events. Generally, eligibility included youth ages 15-24 years, living with HIV (YouThrive) or at-risk for HIV (MyChoices, TechStep, LYNX and P3), who had regular access to a smartphone. A full listing of specific eligibility criteria for each study can be found in Table 1. Participants were consented and enrolled by study recruitment venue staff in-person at the time of the focus group or usability session. Following the National Institutes of Health 2018 Single IRB Policy for Multi-site Research, the University of North Carolina (UNC) institutional review board (IRB) approved all study procedures as the single IRB of record. UNC established institutional reliance agreements with each collaborating recruitment venue's IRB following each institution's standard review procedures.

\section{Data collection}

Data collection occurred between May 2017 and December 2018. Sessions were led by one or two members of each study team, typically a project manager and/or principal investigator. Group facilitators were trained in the conduct of qualitative research methods and focus groups and typically had substantial experience working with youth. Participants first completed a brief survey with demographic and behavioral questions to contextualize the qualitative data. Then, focus groups and usability sessions were conducted in private spaces such as a clinic meeting room or youth center. During focus groups and usability sessions, participants were shown images from in-development prototypes via a PowerPoint presentation, provided study phones with functional prototypes, or could download a test version of the app or other mHealth intervention to their personal devices. All focus groups and usability sessions followed semi-structured interview guides and were audio recorded for transcription and analysis. Following the interview, the facilitator completed a standardized summary form to detail the number of participants, session length, major data themes, and notes on any technical issues.

\section{Analysis}

All sessions were audio recorded and professionally transcribed verbatim. Select transcripts from each study were reviewed for quality control. Analysis was guided by Braun and Clarke's thematic analysis framework (21), which is a systematic process for identifying themes and patterns related to our overarching research question. First, a sample of transcripts from each study were reviewed to identify common words, phrases, sentences, and ideas for the development of a qualitative codebook. The codebook focused on similarities and differences within and across studies and relevant information for adapting subsequent iterations of this research and future mHealth interventions. All transcripts were then independently coded by two members of the iTech Analytic Core in a shared dataset in Dedoose software using open-coding, axial coding, and coding of marginal remarks and comparisons. To assess for intercoder reliability, the two coders double-coded a subset of transcripts and then met to examine the degree to which the coding scheme was consistently applied. A third member of the research team helped resolve inconsistencies across coders and minor changes were made to the codebook to capture emergent themes. Once data were coded, excerpts under specific codes and sub-codes were compiled across all interviews and themes and patterns related to the specific research aims were identified.

\section{Results}

\section{Participant characteristics}

To contextualize the qualitative findings, Table 2 lists the sociodemographic characteristics of participants by study and Table 3 provides information on technology use.

\section{Four main qualitative themes}

Four main themes emerged from our qualitative analysis: (I) there was consistent interest in a range of privacy and confidentiality features centered around a desire to 
Table 1 Overview of data collection methods, location, and characteristics by study

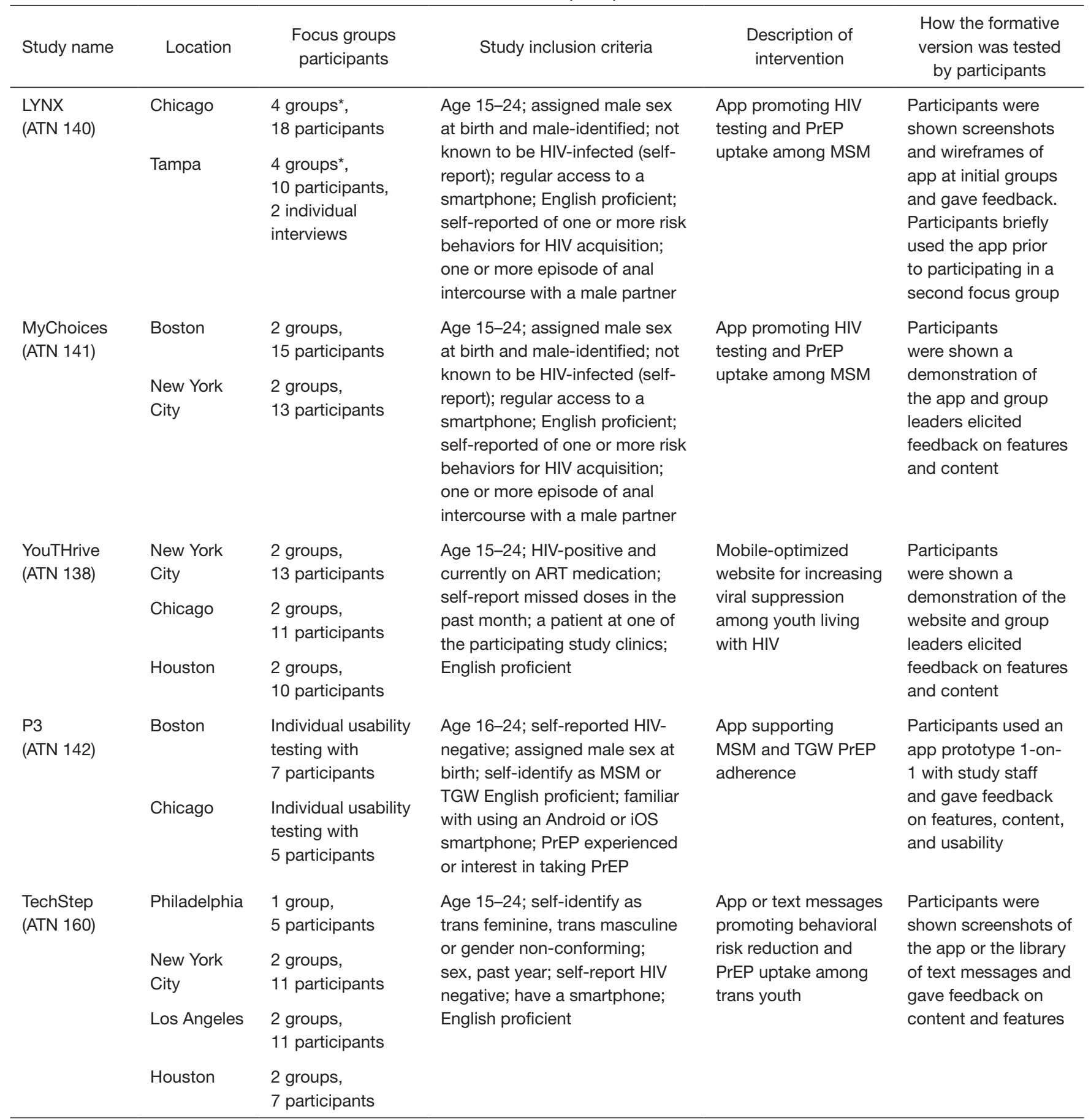

ART, antiretroviral therapy; HIV, human immunodeficiency virus; MSM, men who have sex with men; PrEP, pre-exposure prophylaxis; TGW, transgender women.

avoid stigma; (II) there were significant preferences and sensitivities around the look and feel of interventions in terms of the diversity and identity of the users; (III) across interventions and populations, youth recommended a variety of platform engagement features; and (IV) there were a wide range of content suggestions, which varied by 
Table 2 Participant sociodemographic characteristics

\begin{tabular}{|c|c|c|c|c|c|c|}
\hline Characteristic & Category & $\begin{array}{l}\text { LYNX, } \\
\mathrm{n}[\%]\end{array}$ & $\begin{array}{l}\text { MyChoices, } \\
\mathrm{n}[\%]\end{array}$ & $\begin{array}{l}\text { YouTHrive, } \\
\text { n [\%] }\end{array}$ & $\begin{array}{l}\text { P3, } \\
n[\%]\end{array}$ & $\begin{array}{l}\text { TechStep, } \\
\mathrm{n}[\%]\end{array}$ \\
\hline Total N & - & 30 & 28 & 34 & 12 & 34 \\
\hline Age, years & Mean [range] & 20 [15-24] & 20 [16-24] & 20 [15-24] & $21[17-23]$ & 21 [17-24] \\
\hline \multirow{3}{*}{$\begin{array}{l}\text { Race (not mutually } \\
\text { exclusive) }\end{array}$} & Asian & $4[13]$ & $3[11]$ & 0 & $1[8]$ & $2[6]$ \\
\hline & Other & $6[20]$ & $3[11]$ & $8[24]$ & $2[16]$ & $9[26]$ \\
\hline & $\begin{array}{l}\text { Don't know/prefer not to answer/ } \\
\text { missing }\end{array}$ & $1[3]$ & $2[7]$ & $1[3]$ & 0 & 0 \\
\hline \multirow[t]{2}{*}{ Sex at birth } & Male & $30[100]$ & $28[100]$ & $26[76]$ & $12[100]$ & 9 [26] \\
\hline & Female & 0 & - & $8[24]$ & - & $25[74]$ \\
\hline \multirow[t]{5}{*}{ Gender identity } & Male & 29 [97] & 28 [100] & $23[68]$ & 10 [83] & $2[6]$ \\
\hline & Female & 0 & - & $8[24]$ & 0 & $5[15]$ \\
\hline & Trans female/trans woman & 0 & - & $2[6]$ & $1[8]$ & $4[12]$ \\
\hline & Trans male/trans man & 0 & - & 0 & - & 12 [35] \\
\hline & Genderqueer/gender nonconforming & 0 & - & $1[3]$ & $1[8]$ & $8[24]$ \\
\hline \multirow{3}{*}{$\begin{array}{l}\text { Highest level of } \\
\text { education }\end{array}$} & Some college or technical school & $8[27]$ & $13[46]$ & 9 [26] & $6[50]$ & $20[59]$ \\
\hline & Finished college/tech school or more & $4[13]$ & $3[11]$ & $3[9]$ & $3[25]$ & $4[12]$ \\
\hline & Missing & 0 & 0 & 0 & 0 & $1[3]$ \\
\hline
\end{tabular}

Empty cells indicate category not relevant due to inclusion criteria. GED, General Educational Development.

the intervention's target population.

\section{Protect our privacy and help us avoid stigma}

Stigma related to keeping HIV status, sexual identity, and sexual behavior confidential was consistently discussed. Many participants were sensitive to any mention of HIV/ AIDS or related terminology, even within the passwordprotected interventions. Some youth living with HIV preferred when interventions focused more holistically on health to alleviate stigma and confidentiality concerns, and minimize the psychological burden of being reminded about HIV.

"I think maybe a bit of thought should go into the privacy concern of a notification ... I just wonder if there's a notification that pops up: 'take PrEP right now' and if someone picks up your phone and sees that-maybe there needs to be a different type of setting given." (P3)

"I mean I just feel like the final, the goal of the website for the creators and stuff should definitely be like just to make sure that it makes young people who bave HIV feel like everybody else because 
Table 3 Participant technology use

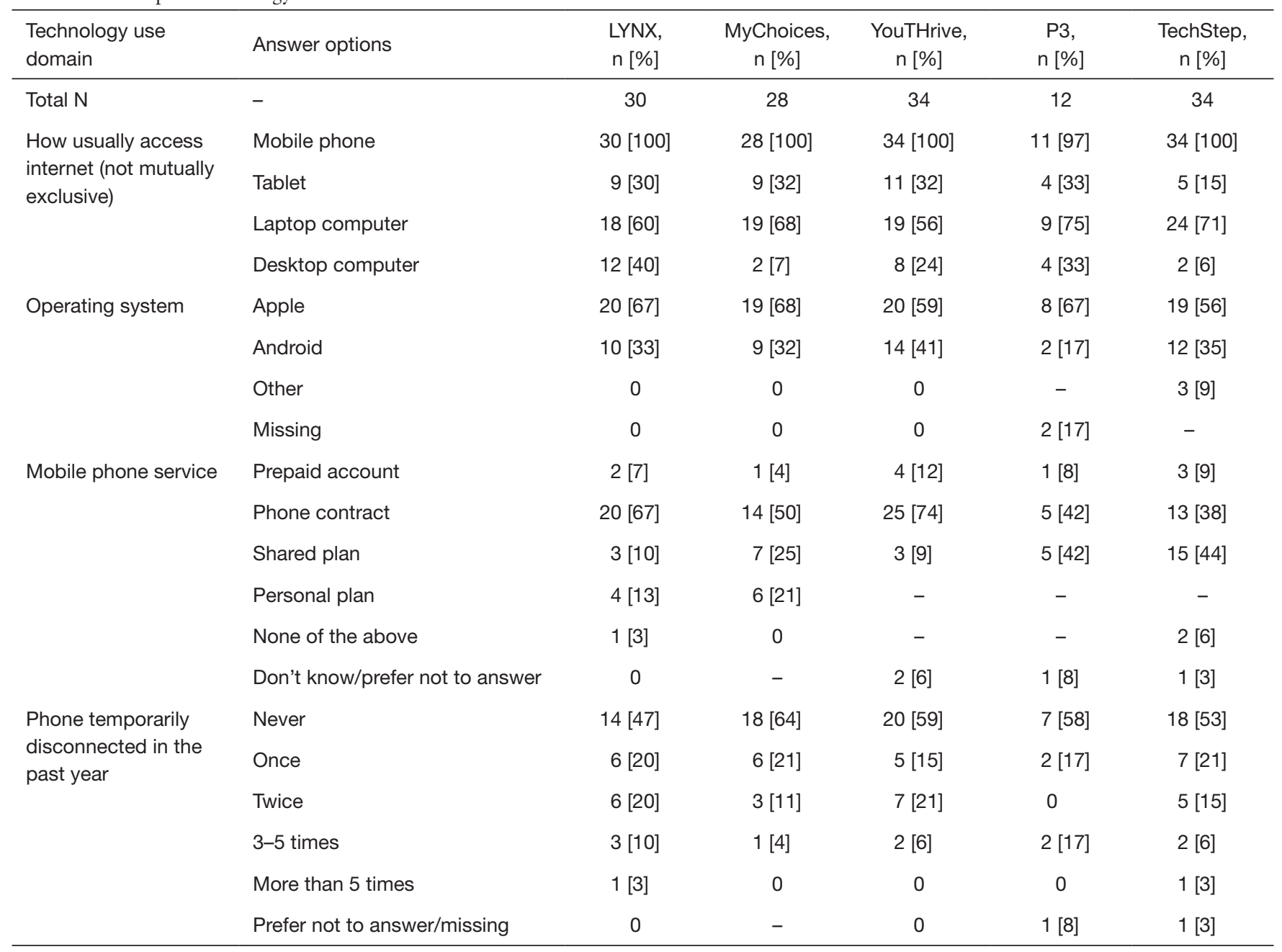

Empty cells indicate answer option not provided.

\section{I think like that's what we all want to feel." (YouTHrive)}

Youth suggested multiple privacy protections on intervention technologies, while also trying to balance ease of access. There was strong support for use of a PIN, password, or touch ID (fingerprint) for entering platforms. Logging in through other platforms (e.g., Facebook, Twitter) was generally not accepted. Auto logout functionality was desired, although timing preference varied from "immediately" to 24 hours. In-app interactions, messaging, and reminders should be discrete and allow for privacy protections.

"Like instead of the log in and log off, [the app] should just always ask [for a passcode] every time you go into the app, and then I would feel comfortable. Because I wouldn't feel comfortable of that if I bave to manually remember to log off or quit the app."
(MyChoices)

"It's kind of like in its own little thing if you don't add the social media to it, like it kind of still makes you feel like you have your own personal privacy." (LYNX)

Participants had mixed perspectives about whether users should be allowed to post identifying photos, share personal contact information, or individually message others within the intervention. Allowing this freedom raised concerns about misuse of the platform (e.g., for hooking up) and privacy, while restricting it raised concerns about limiting social support and further isolating users.

"Well I understand all the - I guess restrictions as far as the messaging and stuff. But I think living with HIV it makes people feel like you just said it makes them feel alone and it already makes you feel in a way-it kind of makes you feel closed off from 
the world already. So, I don't think that all the restrictions and stuff is going to make people feel free." (YouTHrive)

"I like the idea [of the social component of the app] because it kind of builds this community around PrEP when right now I feel like it's kind of like this, secluded, not a lot of people either know about it or there's kind of like a stigma bebind it." (P3)

\section{Respect and reflect our diversity and identity}

Participants across multiple studies valued the ability to customize the look and feel of the platforms, allowing users the ability to change platform themes and colors. A number of youth endorsed features such as avatars, which were seen as a way to personalize the platform while remaining anonymous. Youth emphasized that imagery should be representative of individuals and communities, while still being discrete (e.g., not using gay or transgender icons or colors).

"It's just like when you log on to something and you don't see anything representative of you, you're going to automatically be like, this is probably just-this site is not for me. If you want to get more diverse type groups of people, you want to kind of make it more different than that." (YouTHrive)

"I have friends who are not very politically correct. And if they saw me opening a rainbow app, they would like laugh at me. Like they're fully accepting but they'll just be like, 'that's really cliche' which might mean I may not want to open the app in my own comfort space and whatnot." (MyChoices)

Youth consistently emphasized the importance of positivity, motivational language and themes, and humor. Further, language on the platform should be inclusive and accessible to all users, with sensitivity toward not perpetuating stigma, avoiding academic jargon, and respecting the sexual and gender identity of end users.

"I think the only thing I'd add for you to make it more appealing to millennial or younger audiences is the-like the light quirkiness. Like the reminder responses. Not super gamey but a little flavor and personality is what I see as most useful. But not overdoing it. That's bow you turn me away." (MyChoices)

"[H]ow far can you keep saying 'trans'? Some people might not agree with [that] point of view. So, we should include everybody else, that's why we should take the 'trans' off ... A trans woman like me like one point, was like, 'I'm not trans, I'm a girl'." (TechStep)

Across studies, participants preferred information presented via multiple modalities (e.g., videos, images, tables, and interactive activities) over text-heavy sections. Youth requested more visuals in several parts of the interventions, including substituting videos for text where possible, and providing image-based overviews of intervention features.

"A video would be helpful because I know there are some people out there that would see all of the writing about PrEP and be like, 'I don't want to read all of that'." (MyChoices)

"You need to make it more visual. The information is wonderful. It really is, it just-it needs to be more visual. It needs to draw me in ..." (YouTHrive)

"A walk through or swipe through tutorial when you first log in with the different things like arrows pointing toward you." (TechStep)

\section{Allow us to engage in multiple ways}

Youth preferred engagement features and functions that were consistent with applications and other web-based platforms they use regularly (e.g., Twitter, Facebook, Instagram).

"Would these reminders be integrated with my Apple calendar?... I think if I'd have to go into the app to see that kind of thing, then I'd probably forget about it even then, unless it either comes up as a push notification a while back or it is integrated into my calendar." (MyChoices)

"I imagine that would also look a lot like the code that's used in the Twitter notifications whenever somebody likes multiple things. It just tells you like they like this many tweets and then they allow you to like look into it." (TechStep)

Most youth appreciated a range of gamification features to increase engagement, particularly those that resulted in tangible rewards instead of virtual rewards-e.g., Amazon gift card $v s$. an in-app badge. However, there was support for the badge system in the LYNX app which used humor as its basis. Several youths explained that reward and gamification features were not necessary for a health-related app. Older youth in particular thought that "gamifying health" would not increase platform engagement, but highlighted that preferences may vary by user age and motivations for intervention use.

"If there was maybe some sort of real points or something that you could get like discounts or something like in the real worldlike [an] Amazon gift card or something. I know that's probably not realistic, but I'm very goal oriented with that kind of stuff so that would get me engaged." (P3)

"I think it's a good idea to have badges. I think everyone likes a reward system, a way to like feel like you're doing a good job ... that's going to make you want to work harder and most importantly it's going to make you want to come back to earn more badges." (LYNX)

"I'm more concerned about the content. I don't care about the 
points. I want to know whether this rash is gonna go away, that's all I'm thinking about." (YouTHrive)

Participants strongly desired the ability to customize or personalize intervention engagement features (e.g., intervention messaging, medication reminders, profile images, app color scheme). In regard to intervention messaging and reminder functions in particular, suggestions included providing the capacity to control the frequency (i.e., daily, weekly, monthly), modality (i.e., text, email, push notification), and content of messages.

"I don't know if the app might be able to make smarter choices ... I'm having lunch usually between 12 and two each day. So, could you remind during that time and I'll finally tell you once [I've] taken [my PrEP]. I wonder if this just can be a more customized app." (P3)

"I think you should be able to set [testing reminders] almost like bow you could set a reminder on your phone on like your regular calendar ... where you can set it personally to your own schedule better than having like a regular standard thing that the app generates kind of reads itself because the app doesn't really know your schedule and your lifestyle." (LYNX)

Some youth emphasized the importance of flexibility for in-app interactions (e.g., being able to write out a response message versus clicking an emoji response to a post) and liked the option of interacting with a professional (e.g., study staff member or clinician) through the intervention for timely provision of resources or support.

"I like how that it asks me how I am feeling today, you know. That helps a lot rather than just asking me, 'Hey, you took your meds?' Okay, that's cool, and just like — that helps." (YouTHrive)

"I like the question. The ability to ask a question to a bealth professional. That's a really, really invaluable feature." (MyChoices)

"It'll be good for me because if I miss a dose or, and I'm like freaking out, let's say, I have unprotected sex. And I'm like, I'm not sure what the person I just slept with status is and I forgot to take my PrEP that day. And you're not available to take a STI or HIV test automatically—it's good to have somebody talk to you through it, calm me down, make sure everything's okay." (P3)

\section{Expand available content and resources}

Youth across studies stressed the importance of featuring young role models from the lesbian, gay, bisexual and transgender (LGBT) community, potentially including profiles of people graduating college, successfully living with HIV, racial and ethnic minority LGBT community members, individuals representing diverse gender identities, and trans or queer artists and celebrities. Further, youth suggested providing users with a space to share stories and experiences. Lastly, participants suggested adding information about activism opportunities within the intervention that would allow them to engage with their communities in-person.

"Maybe have like prominent people in the LGBT community that most 22-year-olds with HIV would know and have them have a profile-post them having kids graduating college, doing things that will motivate us and things that will make us feel like everybody else." (YouTHrive)

"What I immediately thought when I saw these pages was like, oh, yeah, a lot of health materials... I feel like what's really missing so far from the app is any sort of, like interaction or representation of the spirit of the community that this app is serving." (MyChoices)

"[More information on] local community orgs because, I am beavily involved in the trans community on my campus. But I'm just like catching glimpses of it off campus." (TechStep)

Many youths wanted intervention content to focus on health more broadly as opposed to only HIV-, HIV risk-, and PrEP-centric content. Suggestions included providing content on healthy relationships, navigating stigma, and seeking healthcare services. Further, there was a consistent desire expressed for more mental health resources (e.g., dealing with depression, suicidality) via these interventions. In addition, youth requested more content about how to talk to partners about PrEP and trans youth requested information on transitioning to adult care and how PrEP may interact with gender affirming hormone therapy.

"If you are going to add anything else, add the mental health services. I mean I know me personally I suffer with some personal mental health issues. And I think it's more prevalent in the gay community and stuff than there is in the other communities." (LYNX)

"Dealing with HIV when you are a student in school ... and depression and anxiety, and what is it like to be a young person with chronic illness dating and disclosure when living with HIV." (YouTHrive)

"I think it would be very helpful, if y'all bad a transitioning topic, because I've had the hardest time transitioning from peds to adult health care." (TechStep)

Maps identifying the locations of youth- and lesbian, gay, bisexual, transgender, queer and/or questioning (LGBTQ)friendly services were a well-received intervention resource. Youth recommended expanding this feature to include a review system where other users could rate services they utilized and allowing more interaction with healthcare providers to facilitate service utilization tracking. 
"Part of me would want a review system in that in case there is a place that's like really bad or that treated someone really poorly ... like wasn't very empathetic to someone who got service there or navigated, for example, gender deviance or their race very - if that wasn't handled well, I would want to know so I don't go to that place and experience similar treatment." (MyChoices)

"I feel like there should be a way for like maybe the healthcare provider, or like when you go get tested or whatever, they can like put your information maybe into the app so they can kind of like say, 'Hey, at this date in this time, you got tested at this place'." (LYNX)

\section{Discussion}

We conducted a cross-cutting analysis of formative research across five samples of sexual and gender minority youth living with or at-risk for HIV, with the objective of informing research aimed at adapting mHealth interventions to best serve youth and inform a best practices model for technology-based intervention development. Similar preferences observed across studies should be incorporated into future iterations of mHealth interventions serving gender and sexual minority youth. This research guiding the rapid construction and adaptation of HIVfocused mHealth interventions for youth is particularly well-timed given an increasing technological capacity to individualize and deliver health interventions (22).

We found consistent feedback regarding intervention privacy protections and customization capabilities. Across studies, participants agreed that interventions should present content and imagery that is inclusive and discrete, and highlighted the importance of the interventions having a positive tone and incorporating accessible (i.e., non-academic) language. Participants also agreed that engagement features and functions should be consistent with familiar platforms (e.g., Instagram). Participants voiced varied preferences regarding the look and feel of interventions, but consistently highlighted the need for customization in these areas.

While this sample of sexual and gender minority youth appreciated-to varying degrees-gamification features and rewards, there were contrasting perspectives on whether gamification features would increase engagement in the intervention and impact health behavior. While gamification features have been shown to increase engagement in various types of non-health specific online programs (23), this finding is consistent with a review of gamification and adherence to web-based mental health interventions, which found no evidence that gamification features improved adherence to intervention programs (24).

Youth had a range of content suggestions, including featuring young, successful role models (e.g., LGBT and minority representation, profiles of successful college graduates, and youth living with HIV or on PrEP); providing a space to share stories; increasing mental health resources; and maintaining a holistic focus on health and relationships. Interest in mental health resources is likely particularly relevant for sexual and gender minority youth, who experience mental health challenges at a significantly higher rate than their cisgender and heterosexual peers (25-27). Among youth living with HIV, disparate psychiatric symptomology can negatively impact HIV treatment outcomes (28-30). Depression, for example, has been shown to negatively impact ART adherence $(29,30)$. Therefore, HIV-focused mHealth platforms serving youth may benefit from the integration of mental health resources.

The range of engagement feature preferences and content suggestions observed in this research suggest that further market segmentation (e.g., youth $<18$, trans youth, etc.) may be needed to best serve the diverse needs of sexual and gender minority youth with respect to the development of HIV prevention and care interventions (31). Further, cross-population differences in preferences indicate where targeted formative work is needed for intervention tailoring within specific population segments. Lastly, while mobile technology has been shown to be an effective means of reaching and engaging youth in health interventions, it is important to note the potential limitations of this approach, including challenges related to access and usability, adequate patient monitoring, and confidentiality (32).

There were several limitations of this cross-study evaluation of formative work. First, participants viewed interventions in various stages of development from early prototypes to fully functional models, and for brief amounts of time prior to critiquing them. Longer-term use of fullydeveloped versions of the interventions may have yielded critiques that were more informed and would more closely reflect an actual participant's experience. Further, qualitative research by design provides in-depth information from smaller sample sizes and is thus not generalizable in the same way as quantitative data. While not necessarily representative, we are confident that our sample of 138 sexual and gender minority youth captures both depth and diversity of perspective by looking across five samples from seven geographically distinct recruitment cities and participants of varying sexual and gender identities and 
HIV statuses. Lastly, our sample may be biased toward individuals who are more likely to be engaged in clinical services and thus not necessarily representative of youth who do not access prevention services and youth living with HIV who are undiagnosed or out of care. Further research is needed to understand the broad applicability of engagement preferences.

\section{Conclusions}

This research reveals challenges in finding engagement features and content that appeal to youth across a wide age range (15-24 years) and with unique health needs. However, several key consistencies in feature preferences identified across studies should serve to inform a core set of best practices for the development of mobile HIV prevention and care interventions for youth. Strategic customization could accommodate variations in visual, function, and feature preferences for future adaptations. However, continued research is needed to optimize intervention customization capabilities to satisfy end users while considering feasibility and minimizing development costs. Further, research on preferences for intervention content among specific segments of youth (e.g., youth $<18$, trans youth, etc.) via youth advisory board feedback or additional formative research will be needed to adapt interventions to best serve end-users. Finally, research is needed to ultimately determine whether preferences correlate with increased engagement and whether that engagement ultimately impacts health outcomes among youth.

\section{Acknowledgments}

Funding: Research reported in this publication was supported by the Eunice Kennedy Shriver National Institute of Child Health and Human Development of the National Institutes of Health under award number U19HD089881 and the National Institute of Mental Health of the National Institutes of Health under Award Number F31MH119965. The content is solely the responsibility of the authors and does not necessarily represent the official views of the National Institutes of Health.

\section{Footnote}

Provenance and Peer Review: This article was commissioned by the Guest Editor (Lisa Hightow-Weidman) for the series

"Technology-based Interventions in HIV Prevention and
Care Continuum among American Youth" published in mHealth. The article has undergone external peer review.

Data Sharing Statement: Available at http://dx.doi. org/10.21037/mhealth-20-43

Peer Review File: Available at http://dx.doi.org/10.21037/ mhealth-20-43

Conflicts of Interest: All authors have completed the ICMJE uniform disclosure form (available at http://dx.doi. org/10.21037/mhealth-20-43). The series "Technologybased Interventions in HIV Prevention and Care Continuum among American Youth" was commissioned by the editorial office without any funding or sponsorship. LHW served as the unpaid Guest Editor of the series. DG, KEM, CH, $\mathrm{KBB}, \mathrm{AYL}, \mathrm{KJH}, \mathrm{JMG}, \mathrm{CJR}$, and LHW report grants from National Institutes of Health, during the conduct of the study; AYL reports grants and other from Gilead Sciences and grants from Viiv Healthcare, outside the submitted work. The authors have no other conflicts of interest to declare.

Ethical Statement: The authors are accountable for all aspects of the work in ensuring that questions related to the accuracy or integrity of any part of the work are appropriately investigated and resolved. The included studies were conducted in accordance with the Declaration of Helsinki (as revised in 2013) and were approved by the Institutional Review Board of the University of North Carolina at Chapel Hill [Office for Human Research Protections Federal Wide Assurance (FWA) \#4801]. Informed consent was obtained for all participants.

Open Access Statement: This is an Open Access article distributed in accordance with the Creative Commons Attribution-NonCommercial-NoDerivs 4.0 International License (CC BY-NC-ND 4.0), which permits the noncommercial replication and distribution of the article with the strict proviso that no changes or edits are made and the original work is properly cited (including links to both the formal publication through the relevant DOI and the license). See: https://creativecommons.org/licenses/by-nc-nd/4.0/.

\section{References}

1. CDC. Youth and HIV. 2019. Available online: https:// www.cdc.gov/hiv/group/age/youth/index.html 
2. Wejnert C, Le B, Rose CE, et al. HIV infection and awareness among men who have sex with men-20 cities, United States, 2008 and 2011. PLoS One 2013;8:e76878.

3. Siegler AJ, Mouhanna F, Giler RM, et al. The prevalence of pre-exposure prophylaxis use and the pre-exposure prophylaxis-to-need ratio in the fourth quarter of 2017 , United States. Ann Epidemiol 2018;28:841-9.

4. Beer L, Mattson CL, Shouse RL, et al. Receipt of clinical and prevention services, clinical outcomes, and sexual risk behaviors among HIV-infected young adults in care in the United States. AIDS Care 2016;28:1166-70.

5. Kahana SY, Fernandez MI, Wilson PA, et al. Rates and correlates of antiretroviral therapy use and virologic suppression among perinatally and behaviorally HIVinfected youth linked to care in the United States. J Acquir Immune Defic Syndr 2015;68:169-77.

6. Zanoni BC, Mayer KH. The adolescent and young adult HIV cascade of care in the United States: exaggerated health disparities. AIDS Patient Care STDS 2014;28:128-35.

7. Kurth AE, Lally MA, Choko AT, et al. HIV testing and linkage to services for youth. J Int AIDS Soc 2015;18:19433.

8. Doll M, Fortenberry JD, Roseland D, et al. Linking $\mathrm{HIV}$-negative youth to prevention services in $12 \mathrm{US}$ cities: barriers and facilitators to implementing the HIV prevention continuum. J Adolesc Health 2018;62:424-33.

9. DiClemente RJ, Sales JM, Borek N. Barriers to adolescents' participation in HIV biomedical prevention research. J Acquir Immune Defic Syndr 2010;54:S12.

10. Hightow-Weidman LB, Muessig K, Rosenberg E, et al. University of North Carolina/Emory Center for Innovative Technology (iTech) for addressing the HIV epidemic among adolescents and young adults in the United States: protocol and rationale for center development. JMIR Res Protoc 2018; 7:e10365.

11. Pew Research Center. Internet and Technology. 2019.

12. Muessig KE, Nekkanti M, Bauermeister J, et al. A systematic review of recent smartphone, Internet and Web 2. 0 interventions to address the HIV continuum of care. Curr HIV/AIDS Rep 2015;12:173-90.

13. Muessig KE, LeGrand S, Horvath KJ, et al. Recent mHealth interventions to support medication adherence among HIV-positive men who have sex with men. Curr Opin HIV AIDS 2017;12:432.

14. Hightow-Weidman LB, Muessig KE, Bauermeister J, et al. Youth, technology, and HIV: recent advances and future directions. Curr HIV/AIDS Rep 2015;12:500-15.

15. Whittaker R, Merry S, Dorey E, et al. A development and evaluation process for mHealth interventions: Examples from New Zealand. J Health Commun 2012;17:11-21.

16. Biello KB, Marrow E, Mimiaga MJ, et al. A mobile-based app (MyChoices) to increase uptake of HIV testing and pre-exposure prophylaxis by young men who have sex with men: protocol for a pilot randomized controlled trial. JMIR Res Protoc 2019;8:e10694.

17. Horvath KJ, Maclehose RF, Martinka A, et al. Connecting Youth and Young Adults to Optimize Antiretroviral Therapy Adherence (YouTHrive): Protocol for a Randomized Controlled Trial. JMIR Res Protoc 2019;8:e11502.

18. Liu A, Coleman K, Bojan K, et al. Developing a mobile app (LYNX) to support linkage to HIV/sexually transmitted infection testing and pre-exposure prophylaxis for young men who have sex with men: protocol for a randomized controlled trial. JMIR Res Protoc 2019;8:e10659.

19. Reback CJ, Rusow, JA, Cain D, et al. Technologybased Stepped Care to Stem Transgender Adolescent Risk Transmission: Study Protocol for a Randomized Controlled Trial (TechStep). JMIR Res Protoc 2020;9:e18326.

20. LeGrand S, Knudtson K, Benkeser D, et al. Testing the efficacy of a social networking gamification app to improve pre-exposure prophylaxis adherence (P3: Prepared, Protected, emPowered): Protocol for a randomized controlled trial. JMIR Res Protoc 2018;7:e10448.

21. Braun V, Clarke V. Using thematic analysis in psychology. Qual Res Psychol 2006;3:77-101.

22. Nahum-Shani I, Smith SN, Spring BJ, et al. Just-intime adaptive interventions (JITAIs) in mobile health: key components and design principles for ongoing health behavior support. Ann Behav Med 2018;52:446-62.

23. Looyestyn J, Kernot J, Boshoff K, et al. Does gamification increase engagement with online programs? A systematic review. PLoS One 2017;12:e0173403.

24. Brown M, O'Neill N, van Woerden H, et al. Gamification and adherence to web-based mental health interventions: a systematic review. JMIR Ment Health 2016;3:e39.

25. Batchelder AW, Safren S, Mitchell AD, et al. Mental health in 2020 for men who have sex with men in the United States. Sex Health 2017;14:59-71

26. Almeida J, Johnson RM, Corliss HL, et al. Emotional distress among LGBT youth: The influence of perceived discrimination based on sexual orientation. J Youth Adolesc 2009;38:1001-14.

27. Connolly MD, Zervos MJ, Barone II CJ, et al. The mental health of transgender youth: Advances in understanding. J 
Adolesc Health 2016;59:489-95.

28. O'Cleirigh C, Magidson JF, Skeer M, et al. Prevalence of Psychiatric and Substance Abuse Symptomatology among HIV-infected Gay and Bisexual Men in HIV Primary Care. Psychosomatics 2015;56:470-8.

29. Hosek SG, Harper GW, Domanico R. Predictors of medication adherence among HIV-infected youth. Psychol Health Med 2005;10:166-79.

30. Uthman OA, Magidson JF, Safren SA, et al. Depression and adherence to antiretroviral therapy in low-, middle-

doi: $10.21037 /$ mhealth-20-43

Cite this article as: Giovenco D, Muessig KE, Horvitz C, Biello KB, Liu AY, Horvath KJ, Golinkoff JM, Reback CJ, Hightow-Weidman L. Adapting technology-based HIV prevention and care interventions for youth: lessons learned across five U.S. Adolescent Trials Network studies. mHealth 2021;7:21. and high-income countries: a systematic review and metaanalysis. Curr HIV/AIDS Rep 2014;11:291-307.

31. Gomez A, Loar R, Kramer AE, et al. Reaching and targeting more effectively: the application of market segmentation to improve HIV prevention programmes. J Int AIDS Soc 2019;22 Suppl 4:e25318.

32. Marcolino MS, Oliveira JA, D'Agostino M, et al. The impact of mHealth interventions: systematic review of systematic reviews. JMIR Mhealth Uhealth 2018;6:e23. 